\title{
A SENSOR AND DATA FUSION ALGORITHM FOR ROAD GRADE ESTIMATION
}

\author{
Per Sahlholm * Henrik Jansson * Ermin Kozica ${ }^{* *}$ \\ Karl Henrik Johansson ** \\ * Scania CV AB, SE-151 87 Södertälje, Sweden \\ ** Royal Institute of Technology (KTH), SE-100 44, \\ Stockholm, Sweden
}

\begin{abstract}
Emerging driver assistance systems, such as look-ahead cruise controllers for heavy duty vehicles, require high precision digital maps. This contribution presents a road grade estimation algorithm for fusion of GPS and vehicle real-time sensor data, with measurements from previous runs over the same road segment. The resulting road grade estimate is thus enhanced using measurements from additional traversals of known roads. Distributed data fusion is utilized to ensure that the storage requirement of known roads does not increase when additional measurements are processed. The implemented algorithm, which is based on extended Kalman filtering and smoothing, is described in detail. Experiments on a Scania test vehicle show the advantages and some of the challenges with the proposed approach.
\end{abstract}

Keywords: Road grade estimation, digital maps, GPS, Kalman filter, sensor fusion

\section{INTRODUCTION}

Several algorithms in today's heavy duty vehicle (HDV) embedded systems are based on state information of the vehicle. Such state estimates are traditionally obtained from one or more sensors in the vehicle. In addition to the traditional applications new advanced driver assistance systems under development predict the future dynamics of the vehicle. These predictions combine the current state of the vehicle with, stored or sensed, information about the road ahead e.g., the topology, the curvature or the traffic situation.

Prediction of the behavior of the vehicle over significant distances requires high quality map data. Highway speed optimization for heavy duty vehicles often requires prediction of the vehicle dynamics for the next kilometer or more. The problem essentially becomes open loop since the control decisions have to be made before any conclusive feedback is available. Some domain knowledge about road construction may be applied, but high quality maps with road grade information will still be needed.

The maps can either be bought or obtained by own measurements. In order to obtain adequate data quality from a single drive over the road it is necessary to use sophisticated measurement equipment. Combining sensor fusion, based on several ordinary sensors, and data fusion of multiple measurement runs obtained for the same road segment, it is possible to approach the required quality level. This contribution considers a system for HDVs suitable for an optimizing cruise control. HDVs often travel the same highways frequently, and may thus collect overlapping data sequences from several measurements within a reasonable time frame. 
Many current vehicles include a GPS sensor of some kind, and it is foreseen that the market penetration for Global Navigation Satellite Systems (GNSS) will increase substantially in the coming decade. Future standard sensors can also be expected to offer enhanced performance. This contribution is focused on describing and evaluating the performance of a sensor and data fusion road grade estimation system based on standard mounted HDV sensors and an additional GPS sensor.

\subsection{Related Work}

How knowledge about the upcoming road topology can be used for optimizing the vehicle's speed profile with respect to fuel consumption has been presented in several recent contributions e.g., (Lattemann et al., 2004; Terwen et al., 2004; Fröberg et al., 2006; Hellström et al., 2006; Hellström et al., 2007; Fröberg and Nielsen, 2007). In this line of work knowledge about the road grade ahead of the truck is assumed to be known e.g., via a map. As previously mentioned, this contribution considers the task of developing a system for creating road grade maps for this type of application. The idea is to create a system that merges sensor data from several data sequences measured on a road segment into a map.

Similar ideas have been presented by Schroedel et. al. (Schroedl et al., 2004) and Brüntrup et. al. (Brüntrup et al., 2005) where data mining is used to automatically create road network maps from a (large) collection of individual GPS traces. Neither of these sources however explicitly address road grade estimation or the possibility to use a vehicle model to improve estimation quality.

There are several different methods proposed in the literature for estimation of the road grade. One way is to use a sensor that is directly related to the grade. Such a solution is presented by Bae et al (Bae et al., 2001) where the grade is determined using a GPS receiver by calculating the ratio between the vehicle's vertical velocity and its horizontal velocity. A GPS receiver needs good satellite coverage to obtain decent estimates. This is however, a constraint that is difficult to sustain in areas with buildings, trees, tunnels or other large objects. There are also methods that recursively estimates the road grade without using direct sensor information. Lingman and Schmidtbauer (Lingman and Schmidtbauer, 2001) presents a method where the road grade is estimated using a Kalman filter-based on measured or estimated propulsion force, estimated retardation forces and measured velocity. Vahidi et. al. (Vahidi et al., 2005) presents a similar method where the grade is estimated using Recursive
Least Squares-based on a simple motion model. An advantage with these methods is that no extra sensors are required. There are however certain occasions when these two methods fail, or have major difficulties, to deliver reliable estimates e.g., when the friction brakes are applied or when gearshifts are performed.

\subsection{Contribution}

This paper presents a novel method to estimate the road grade-based on standard mounted HDV sensors and a GPS unit. A major contribution is the spatial sampling of the sensor fusion estimate which through the estimate error covariance matrix enables data fusion of an arbitrary number of measurement series at difference time instants. The only information that needs to be retained for each sample point in addition to the state estimate is the error covariance matrix estimate. Using this method map data storage requirements for a fixed road will not grow when additional measurements are added. The method is able to work reliably even if either the GPS data or the vehicle model data temporarily becomes unavailable. This could happen due to signal masking or brake application. If both signals are lost simultaneously the error covariance estimate will be large, and the section will not have significant impact on already stored map data. The results obtained from the described method are compared to independently collected road grade data sequences from a specialized measurement vehicle.

\subsection{Outline}

Models and measurements utilized in the filter are described in Section 2. Section 3 gives a review of the used filtering methods together with a description of how the covariance matrices for the filter are chosen. Section 4 shows results where the road grade estimation algorithm has been applied on data collected during a drive on a Swedish highway. The paper is concluded in Section 5

\section{MODELS AND MEASUREMENTS}

This section describes the models and measurements used for road grade estimation.

\subsection{Models}

In order to do model-based sensor fusion it is necessary to establish some basic relationships between the various signals that are available for measurement and the quantities to be estimated. 


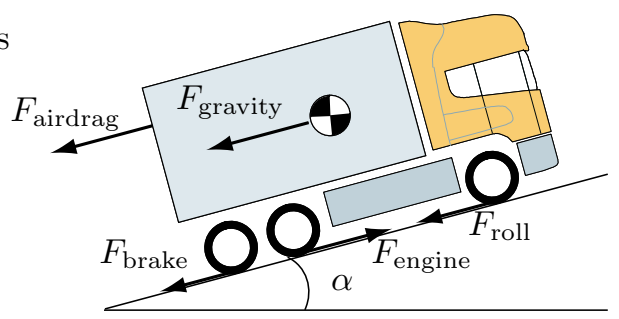

Fig. 1. Longitudinal forces acting on the vehicle.

The model is divided into two parts. The first one describes the longitudinal movement of the vehicle. The second part describes the topology of the road by relating the altitude with the grade and the speed of the vehicle.

Information about the road grade can be obtained from the engine loading and velocity behavior of the vehicle. A principal sketch of the longitudinal forces acting on a HDV is shown in Figure 1. $F_{\text {engine }}$ is the net pull force produced by the engine, $F_{\text {brake }}$ is the applied brake force, $F_{\text {airdrag }}$ is the air drag, $F_{\text {roll }}$ is the rolling resistance and $F_{\text {gravity }}$ is the gravity induced force. Using Newton's law of motion the force balance for the $\mathrm{HDV}$ in Figure 1 is given by

$m_{\mathrm{t}} \dot{v}=F_{\text {engine }}-F_{\text {brake }}-F_{\text {airdrag }}-F_{\text {roll }}-F_{\text {gravity }}$

where $m_{\mathrm{t}}$ is the total accelerated mass, $v$ is the velocity and $\alpha$ denotes the road grade. The longitudinal forces are given by

$$
\begin{aligned}
F_{\text {engine }} & =\frac{i_{\mathrm{t}} i_{\mathrm{f}} \eta_{\mathrm{t}} \eta_{\mathrm{f}}}{r_{\mathrm{w}}} M \\
F_{\text {airdrag }} & =\frac{1}{2} c_{\mathrm{w}} A_{\mathrm{a}} \rho_{\mathrm{a}} v^{2} \\
F_{\text {roll }} & =m g c_{\mathrm{r}} \\
F_{\text {gravity }} & =m g \sin \alpha
\end{aligned}
$$

where $r_{\mathrm{w}}$ is the wheel radius. The transmission and final drive gear ratios $i_{\mathrm{t}}$ and $i_{\mathrm{f}}$, and the corresponding efficiencies $\eta_{\mathrm{t}}$ and $\eta_{\mathrm{f}}$ are vehicle parameters. The net engine torque output $M$ is measured. $c_{\mathrm{w}}$ is the air drag coefficient, $A_{\mathrm{a}}$ is the vehicle front area and $\rho_{\mathrm{a}}$ is the air density. Gravity is denoted by $g$ and $m$ is the vehicle mass. $c_{\mathrm{r}}$ is a coefficient of rolling resistance. The braking torque $F_{\text {brake }}$ is hard to measure or model, and it is most often zero, therefore it is excluded from the model from now on. Considering also engine inertia $J_{\mathrm{e}}$ and wheel inertia $J_{\mathrm{w}}$ in addition to the vehicle mass, and assuming no $\operatorname{slip}\left(v=r_{\mathrm{w}} \omega_{\mathrm{w}}\right)$, the total accelerated mass is

$$
m_{\mathrm{t}}=\frac{J_{\mathrm{w}}}{r_{\mathrm{w}}^{2}}+m+\frac{i_{\mathrm{t}}^{2} i_{\mathrm{f}}^{2} \eta_{\mathrm{t}} \eta_{\mathrm{f}} J_{\mathrm{e}}}{r_{\mathrm{w}}^{2}} .
$$

Equations (1) and (2) can be combined into

$$
\begin{aligned}
\dot{v} & =\frac{r_{\mathrm{w}}^{2}}{J_{\mathrm{w}}+m r_{\mathrm{w}}^{2}+i_{\mathrm{t}}^{2} i_{\mathrm{f}}^{2} \eta_{\mathrm{t}} \eta_{\mathrm{f}} J_{\mathrm{e}}}\left(F_{\text {engine }}\right. \\
& \left.-F_{\text {airdrag }}-F_{\text {roll }}-F_{\text {gravity }}\right)
\end{aligned}
$$

Details of this model can be found in (Kiencke and Nielsen, 2003)

Two states are used to describe the topology of the road, the altitude $z$ and the grade $\alpha$. The dynamics for these two states are

$$
\begin{aligned}
\dot{z}(t) & =v(t) \sin \alpha(t) \\
\dot{\alpha}(t) & =0
\end{aligned}
$$

Since the data fusion method used utilizes spatially sampled measurements the time domain relations (3) and (4) have to be expressed in the spatial domain instead. This can be achieved by replacing the independent variable $t$ with the distance $s$, and using the following relation

$$
\frac{\partial v(t)}{\partial t}=\frac{\partial v(t)}{\partial s(t)} \underbrace{\frac{\partial s(t)}{\partial t}}_{v(t)} .
$$

A combination of the spatial versions of (3)-(4) together with a first order Euler approximation yields a discrete spatially sampled model with sampling distance $\Delta s$ as

$$
\underbrace{\left[\begin{array}{c}
v_{k} \\
z_{k} \\
\alpha_{k}
\end{array}\right]}_{x_{k}}=\underbrace{\left[\begin{array}{c}
v_{k-1}+\Delta s \Delta v_{k} \\
z_{k-1}+\Delta s \sin \alpha_{k-1} \\
\alpha_{k-1}
\end{array}\right]}_{f\left(x_{k-1}\right)}+\underbrace{\left[\begin{array}{c}
w_{k}^{v} \\
w_{k}^{h} \\
w_{k}^{\alpha}
\end{array}\right]}_{w_{k}}
$$

where process noise $w_{k}$ has been added to capture the uncertainty in the model. Subscript $k$ denotes the discrete sample number. The change in velocity $\Delta v_{k}$ during the travel from the previous sample point is given by

$\Delta v_{k}=c_{1} \frac{M_{k-1}}{v_{k-1}}-c_{2} v_{k-1}-c_{3} \frac{1}{v_{k-1}}-c_{4} \frac{\sin \left(\alpha_{k-1}\right)}{v_{k-1}}$

where $c_{1}, \ldots, c_{4}$ are vehicle parameters.

\subsection{Measurements}

This section describes the measured quantities and their relation to the states in (5). A data sequence from a measurement run on a typical Swedish highway is shown in Figure 2.

The GPS is used to record latitude, longitude, traveled distance, altitude and the number of active satellites. The latitude, longitude and traveled distance signals are used to resample the recorded measurements from the original time indexed to a distance indexed form. The altitude signal is used in the road grade estimation with the following sensor model

$$
z_{k}^{\mathrm{GPS}}=z_{k}+e_{k}^{\mathrm{zGPS}} \text {. }
$$

Here $e_{k}^{z \text { GPS }}$ is used to represent stochastic measurement noise.

From standard internal sensors in the vehicle the velocity, net engine torque, brake system usage, 


\section{ROAD GRADE ESTIMATION}
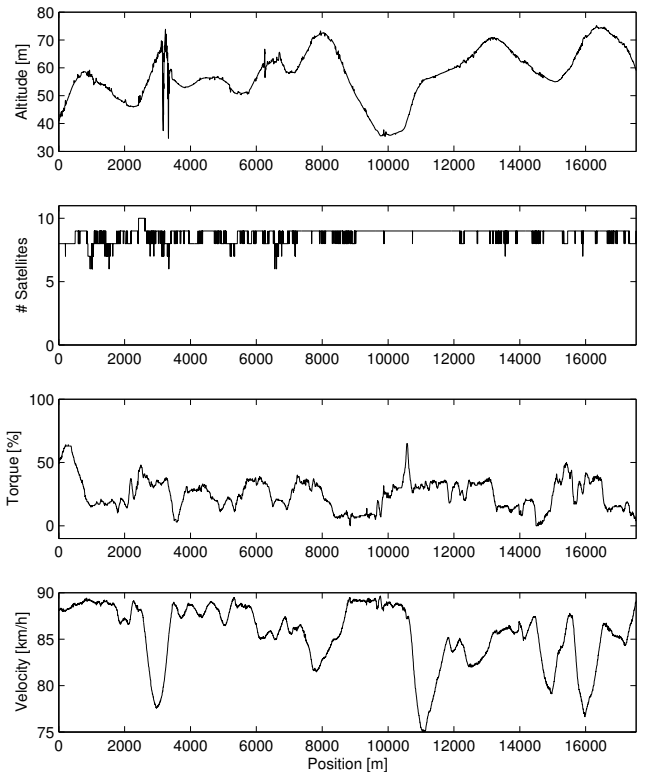

Fig. 2. Example source data sequence measured on a segment of highway E4 south of Södertälje. The first plot shows the GPS altitude signal. The second plot shows the number of active satellites. Plot number three shows the engine torque. The bottom plot shows the measured mean front axle speed.

current gear and gear shifts are recorded. The vehicle velocity is denoted $v^{\text {veh }}$ and is obtained either from the tachograph or the wheel speed sensors. These sensors count the number of revolutions per time unit of either a wheel or the output shaft from the gear box. This gives a rotational speed that is scaled with an applicable gear ratio and wheel radius to obtain a corresponding road velocity of the vehicle. There is typically an offset in this scaling factor. One reason for this is that the wheel radius varies over time due to load, wear or differences in tire pressure. We will, due to space limitations, assume this offset known, and discard estimation of this quantity in this contribution. The sensor model for the vehicle velocity is given by

$$
v_{k}^{\mathrm{veh}}=v_{k}+\delta_{k}^{\mathrm{v}}+e_{k}^{\mathrm{vveh}}
$$

where $\delta_{k}^{\mathrm{v}}$ is due to the offset in the scaling factor and $e_{k}^{\text {vveh }}$ is the measurement noise.

By combining (6) and (7), the relation between the measurements and the states is given by

$$
y_{k}=\left[\begin{array}{c}
v_{k}^{\mathrm{veh}} \\
z_{k}^{\mathrm{GPS}}
\end{array}\right]=\underbrace{\left[\begin{array}{c}
v_{k}+\delta_{k}^{\mathrm{v}} \\
z_{k}
\end{array}\right]}_{h\left(x_{k}\right)}+\underbrace{\left[\begin{array}{c}
e_{k}^{\mathrm{vveh}} \\
e_{k}^{\mathrm{zGPS}}
\end{array}\right]}_{e_{k}} .
$$

Combining the previous sections the measurements and the state update are described by the state-space system

$$
\begin{aligned}
& x_{k}=f\left(x_{k-1}\right)+w_{k} \\
& y_{k}=h\left(x_{k}\right)+e_{k}
\end{aligned}
$$

cf., (5) and (8). It is assumed that the noise sources $w_{k}$ and $e_{k}$ can be represented by zeromean white Gaussian noise processes. With this assumption, extended Kalman filtering (Haykin, 2001) provides a method for estimation of the state vector $x_{k}$ based on the measurements $y_{k}$. This method will be used to create an initial estimate of the road grade for each measurement run. The extended Kalman filter is defined by the system equations (9) together with the covariances for $w_{k}$ and $e_{k}$. Section 3.1 describes the filter recursions in extended Kalman filtering. The considered application suggests that the data processing can be completed with some time delay. It is then useful to create an acausal filter to compensate for the filtering delay and utilize data from future sample points. Such a smoothing algorithm is described in Section 3.2. The noise covariances are tuning variables in the filter that can be adjusted to describe the reliability of different parts of the system or the measurement equations. This reliability typically changes over time and it is therefore very useful to use time-varying covariances. The choice of covariance matrices for road grade estimation is discussed in Section 3.3. To fully utilize the information contained in several measurements a data fusion scheme is required. This step is described in Section 3.4. An overview of the data flow in the proposed method is given in Figure 3

\subsection{Extended Kalman Filtering}

In extended Kalman filtering the non-linear system is linearized around the current trajectory. The standard recursions for Kalman filtering are then applied on the linearized system. These recursions are described by two update steps: a time update and a measurement update. In the first step, the time update, the state estimate $\hat{x}_{k-1}$ and the error covariance $P_{k-1}$ are updated according to

$$
\begin{aligned}
F_{k} & :=\frac{\partial f}{\partial x}\left(\hat{x}_{k-1}\right) \\
\hat{x}_{k} & :=F_{k} \hat{x}_{k-1} \\
P_{k} & :=F_{k} P_{k-1} F_{k}^{T}+Q_{k}
\end{aligned}
$$

where $Q_{k}$ is the covariance of the process noise $w_{k}$. For a stationary noise process $w_{k}$, the covariance matrix is set to a constant in the extended Kalman filter: $Q_{k}=Q$. Here $Q_{k}$ is updated according to 


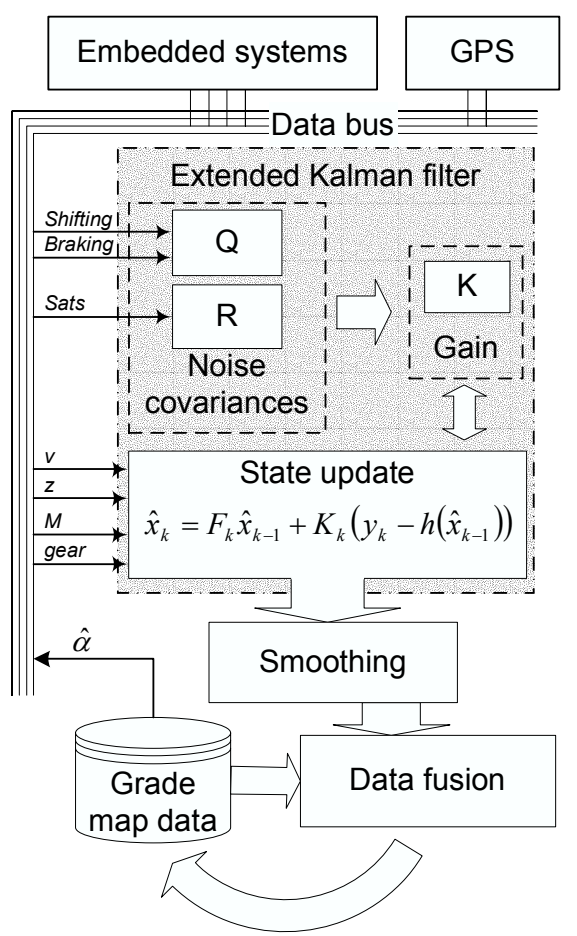

Fig. 3. Overview of filter architecture for road grade estimation. Information from the vehicle's data bus is integrated using an extended Kalman filter, followed by a smoothing algorithm. The smoothed data is then merged with pre-existing data sequences. Information is delivered to the data bus from on-board embedded systems and a GPS-receiver. The grade estimate is denoted $\hat{\alpha}$.

how well different driving conditions are described by the vehicle model. How the piece-wise constant matrix $Q_{k}$ is updated and at which events is further described in Section 3.2

The second step is a measurement update where the estimate is corrected based on the measurements according to

$$
\begin{aligned}
H_{k} & :=\frac{\partial h}{\partial x}\left(\hat{x}_{k}\right) \\
K_{k} & :=P_{k} H_{k}^{T}\left(H_{k} P_{k} H_{k}^{T}+R_{k}\right)^{-1} \\
\hat{x}_{k} & :=\hat{x}_{k}+K_{k}\left(y_{k}-h\left(\hat{x}_{k}\right)\right) \\
P_{k} & :=P_{k}-K_{k} H_{k} P_{k}
\end{aligned}
$$

where $R_{k}$ is the covariance of the measurement noise $e_{k}$. The covariance of the matrix $R_{k}$ is updated at discrete events similar to $Q_{k}$.

\subsection{Smoothing}

By completing the measurement of a road segment before application of the sensor and data fusion, smoothing can be used to compensate for the filtering delay and include information from future sampling points in each estimate. In this work the Rauch-Tung-Striebel fixed point smoothing algorithm has been used. The algorithm is more
Table 1. Recorded events which are taken into account when determining the time varying process noise covariance $\left(Q_{k}\right)$ and measurement noise covariance $\left(R_{k}\right)$.

\begin{tabular}{ll}
\hline Event & Effect \\
\hline Friction brake use & Driveline torque unknown \\
Auxiliary brake use & Uncertain driveline torque \\
Gear shifts & Unmodelled driveline dynamics \\
GPS signal masking & No or low quality GPS data \\
\hline
\end{tabular}

extensively discussed in for example (Kailath et al., 2000).

The smoothing is applied as a backwards recursion when the complete filtered estimate for a road segment has been collected. The filtered estimates $\hat{x}_{k}$ and the estimated error covariance matrices $P_{k}$ are used in the process. The smoothed state estimates $\hat{x}_{k}^{\mathrm{s}}$ and corresponding smoothed estimated error covariance matrices $P_{k}^{\mathrm{s}}$ are later used in the data fusion step of the grade estimation method described in Section 3.4. The final state of the filter recursion is used as initialization for the smoothing backwards recursion

$$
\begin{aligned}
F_{k}^{\mathrm{s}} & :=P_{k} F_{k}^{T} P_{k+1}^{-1} \\
\hat{x}_{k}^{\mathrm{s}} & :=\hat{x}_{k}+F_{k}^{\mathrm{s}}\left(\hat{x}_{k+1}^{\mathrm{s}}-\hat{x}_{k+1}\right) \\
P_{k}^{\mathrm{s}} & :=P_{k}+F_{k}^{\mathrm{s}}\left(P_{k+1}^{\mathrm{s}}-P_{k+1}\right) F_{k}^{\mathrm{s} T} .
\end{aligned}
$$

\subsection{Covariance Matrices}

The implementation is now straightforward, with the exception of choosing noise covariances. As described earlier, the time-varying covariance matrices $Q_{k}$ and $R_{k}$ are chosen to give the filter a desired behavior. This section describes one way for choosing the update of these covariances, specific to road grade estimation.

To simply the design it is assumed that the covariance matrices are diagonal. To handle certain events e.g., braking (which change the system characteristics in (5) and (8)), the covariance matrices are made dependent of such events. The considered events are listed in Table 1. For detection of these events the recorded signals from the vehicle, described in Section 2.2, are used. The estimated time varying error covariance $P_{k}$ will contain confidence information for estimate at each sample point. This information is useful in the data fusion described in Section 3.4.

Signal masking in the GPS-receiver, i.e. reduced number of available satellites due to obstacles in the vicinity has severe effects on the quality of the measurements of the GPS-receiver. Especially the precision of the altitude estimate is dependent on the number available satellites (and also their relative positions, although this effect is not considered here). Thus the size of the variance of $e^{\mathrm{zGPS}}$ 
can be varied according to the current number of available satellites e.g., being inverse proportional to this number. Furthermore, when the satellite count drops below four altitude determination is not possible with GPS, and a very high variance is set. In this way the estimate will not depend on corrupt GPS measurements during periods with low satellite coverage.

A similar reasoning can be applied for braking and shifting, with the addition that these affect the vehicle behavior and thus the process noise as well. It is difficult to estimate the brake force that acts on the vehicle. As a consequence, the equation for the longitudinal dynamics (3) becomes uncertain. A way to handle this is to increase the process noise $w_{k}^{\mathrm{v}}$, whenever the friction brakes are applied. Using wheel rotation to determine the vehicle speed also becomes less reliable during braking, since the amount of slip changes and even lockups can occur. During gear shifts the produced torques in the driveline are difficult to model. Oscillations which are not included in the relatively simple model (3) can be introduced.

The physical relationship between the altitude and the slope suggests an approximate relationship between their process covariances. For the rest of the entries an educated guess based on known sensor characteristics and logged data sequences, yielding desirable filter performance has to be made.

\subsection{Data Fusion}

There are several possible approaches to merging overlapping data sequences from multiple runs. One large filter could be constructed, which takes all measurements into account when estimating a road grade profile. Such an approach would require all measurement data to be saved and reprocessed after a new measurement run.

In this work a distributed Kalman filter is used to incrementally create a total estimate based on one estimate from previous measurements and one new measurement run. This limits the storage requirement to the current set of state estimates and their estimated error covariances. When a new measurement sequence becomes available it is be used to find a new estimate of the grade profile, with an associated error covariance, using the general fusion formula described in e.g., (Gustafsson, 2000)

$$
\begin{aligned}
& P_{k}^{\mathrm{f}}:=\left(\left(P_{k}^{1}\right)^{-1}+\left(P_{k}^{2}\right)^{-1}\right)^{-1} \\
& \hat{x}_{k}^{\mathrm{f}}:=P_{k}^{\mathrm{f}}\left(\left(P_{k}^{1}\right)^{-1} \hat{x}_{k}^{1}+\left(P_{k}^{2}\right)^{-1} \hat{x}_{k}^{2}\right) .
\end{aligned}
$$

Here $\hat{x}_{k}^{\mathrm{f}}, P_{k}^{\mathrm{f}}$ denotes the resulting fused state and error covariance, and $\hat{x}_{k}^{1}, P_{k}^{1}$ and $\hat{x}_{k}^{2}, P_{k}^{2}$ denote the source quantities. Two of the states used in the road grade estimation, $z_{k}$ and $\alpha_{k}$, are directly related to the road. The third state, $v_{k}$, describes the measurement vehicle, and is not constant between measurements. Only the states describing the road are used in the data fusion process, giving $\hat{x}_{k}^{\mathrm{f}}=\left[\begin{array}{ll}\hat{z}_{k}^{\mathrm{f}} & \hat{\alpha}_{k}^{\mathrm{f}}\end{array}\right]^{T}$. If two overlapping data sequences are being merged $\hat{x}_{k}^{1}, P_{k}^{1}$ and $\hat{x}_{k}^{2}, P_{k}^{2}$ consist of the relevant elements of the smoothed estimates $\hat{x}_{k}^{\mathrm{s}}$ and $P_{k}^{\mathrm{s}}$. If a new measurement is merged with an existing map (based on two or more previously merged overlapping data sequences) one of the source estimates is the smoothed new measurement data, and the other source estimate is the map.

The estimated error covariance of the fused estimate will be lower than that of any of the source data sets. If the errors in different measurement runs are not independent as assumed, the fused $P_{k}^{\mathrm{f}}$ will be an underestimate. Segments of each source estimate with high error covariance estimates will have less weight in the calculation. As a result measurements obtained during periods of braking or loss of satellite coverage in one of the data sequences will not destroy higher quality information in the other. A problem to consider is that inherently difficult sections, such as downhill tunnel segments will still be very hard to estimate accurately, since any measurement will most likely contain low-quality estimates.

\section{EXPERIMENTAL RESULTS}

In this section we will present results where extended Kalman filtering as described in Section 3 has been applied to data sequences collected during a test drive. The experimental setup is described in Section 4.1 and the experimental results are presented in Section 4.2.

\subsection{Experimental Setup}

The experiments have been performed with a Scania R420 tractor. All the data have been collected from the vehicle's CAN bus, except for the GPS data. The measurement vehicle is equipped with a GPS, but it was not used, since it does not provide a CAN interface.

The used receiver was a Racelogic VBOX III GPS, and it was connected to the measurement computer together with the vehicle CAN bus through a common CAN interface. A data sequence measured during a drive on a typical Swedish highway is shown in Figure 2. The objective is to use multiple such measurements to estimate the road profile. 


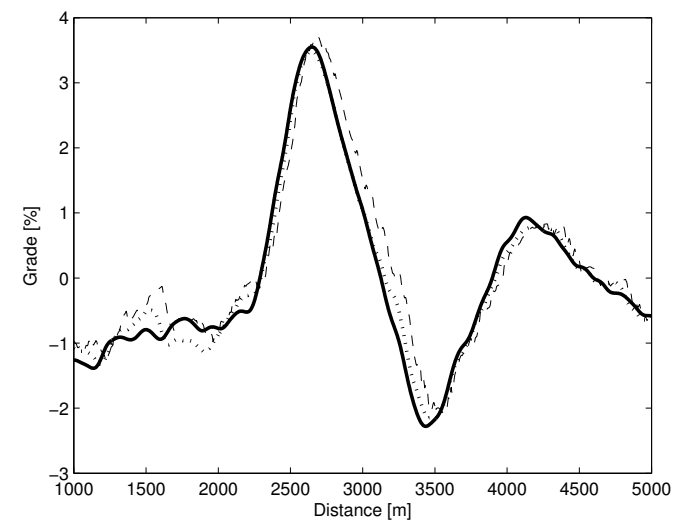

Fig. 4. The filtered (dashed) and smoothed (dotted) grade estimates are shown together with a reference data sequence (solid). The smoothed estimate clearly deviates less from the reference. The lack of filtering delay in the smoothed estimate is an important part of the enhancement.

\subsection{Results}

The proposed road grade estimation method has been applied on data sequences collected from the Swedish highway E4 from Södertälje to Nyköping. So far two measurements have been combined using the extended Kalman filtering, RTS smoothing and distributed data fusion method described.

When estimation results are compared to independent data collected with a specialized measurement vehicle the benefit of the smoothing step can be clearly seen. Figure 4 shows the filtered and smoothed road grade estimates for a road segment together with the reference data sequence.

While single measurements can suffer from uncertainties due to GPS reception issues, braking, gearshifting etc. the combined result from many measurement should be more reliable.

Estimated error covariances for the road grade state, the estimated road grade, the data fusion weights for the measurements and the number of active satellites at each sample point are shown in Figure 5. Fewer satellites gives a higher error covariance estimate, and thus a lower weight in the data fusion step. However, since the used measurements are from a highway with good GPS reception and favorable conditions they receive almost equal weight.

\section{CONCLUSIONS}

The proposed method used on overlapping vehicle and GPS data sequences can produce results which are very similar to single pass reference measurements using a specialized measurement
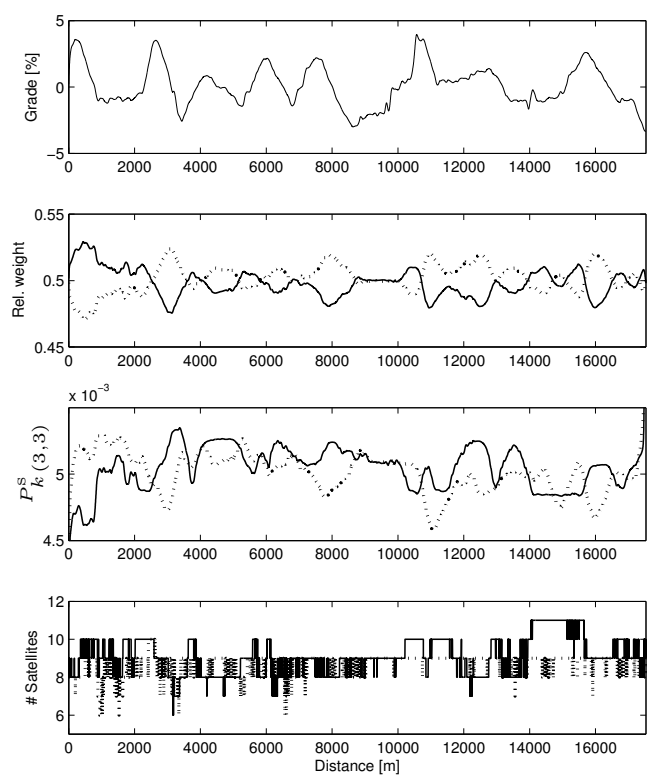

Fig. 5. The first plot shows the final calculated road grade estimate for two measurements. The second plot shows the weight factors in the data fusion step for the first (solid) and second (dashed) measurement. The third plot shows the $(3,3)$ element (grade estimate error covariance) of the estimated smoothed state error covariance matrices for the two measurements. The last plot shows the number of satellites available during the measurements.

vehicle. Multiple measurement runs can help offset GPS signal disturbances that are not directly related to limited coverage in one area. The use of a vehicle model in the filter effectively attenuates high frequency noise from the GPS altitude signal. The vehicle model also reduces the influence of physically unrealistic level changes in the GPS signal on the grade estimate.

The GPS altitude information on the other hand reduces the appearance of a grade estimate bias from modeling or model parameter errors. A comparison of estimated road grade and altitude profiles obtained with the GPS enabled and disabled is shown in Figure 6. The filter without GPS information is initialized at the same altitude as the GPS filter in order to make the comparison.

The scheme to vary the process noise and measurement error covariance matrices depending on additional information from the measurement allows the filter to use the best information from multiple overlapping data sequences for estimating a particular road section. The performance of the algorithm depends on the accuracy of the altitude measurement of the GPS. An area of future work is to compare the performance when using a standard quality vehicle mounted GPS to 

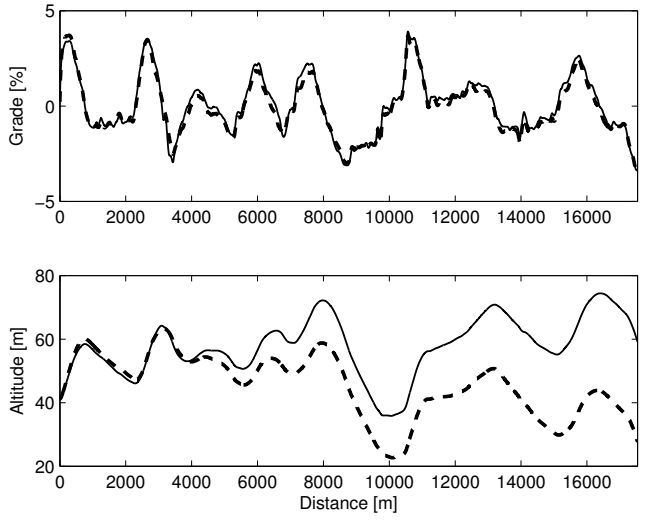

Fig. 6. The first plot shows the road grade estimates for with (solid) and without (dashed) the GPS enabled. Without the GPS a slight model or parameter error causes an underestimate of the road grade. The second plot verifies the conclusion by showing the estimated altitude with (solid) and without (dashed) GPS enabled.

the GPS unit used here. Convergence properties for the fused data, when using sensors of varying quality, are also of great interest. The method should also be further tested on more challenging data sets, with higher noise

As more and more roads are added the error covariance estimate of the database may decay to a level where new information only has marginal impact. This will be a problem in case of changes to the actual road profile. In order to build a complete system for online map creation this problem will have to be addressed.

In order to use the presented methods each measurement has to be synchronized to the others in such a way that the used measurement points represent common real world locations. Using the high frequency sampled raw GPS data sequences finding a common starting point with adequate precision is straightforward. From this point the GPS traveled distance measurement is used to index and resample the data sequence at even spacings. This approach works as long as the vehicle paths from the two runs are close, and the measurement error in the traveled distance is small. Experience shows that 10-15 km long highway road profiles can be used. To enable online map creation, it is necessary to solve this issue, and it is a topic for future work.

\section{ACKNOWLEDGEMENTS}

This work has been partially supported by Scania CV AB, the Swedish Program Board for Automotive Research and the European Commission through the Network of Excellence HYCON.

\section{REFERENCES}

Bae, H. S., J. Ruy and J. Gerdes (2001). Road grade and vehicle parameter estimation for longitudinal control using GPS. In: Proceedings of IEEE Conference on Intelligent Transportation Systems. San Francisco, CA.

Brüntrup, R., S. Edelkamp, S. Jabbar and B. Scholz (2005). Incremental map generation with GPS Traces. In: Proceedings of IEEE Intelligent Transportation Systems. Vienna, Austria.

Fröberg, A. and L. Nielsen (2007). Fuel optimal speed profiles. Accepted to 5th IFAC Symposium on Advances in Automotive Control. Monterey Coast CA, USA.

Fröberg, Anders, Erik Hellström and Lars Nielsen (2006). Explicit fuel optimal speed profiles for heavy trucks on a set of topographic road profiles. number 2006-01-1071 In: SAE World Congress 2006.

Gustafsson, F. (2000). Adaptive filtering and change detection. John Wiley \& Sons, LTD, Chichester.

Haykin, S. (2001). Adaptive Filter Theory. Prentice Hall.

Hellström, E., M. Ivarsson, J. Åslund and L. Nielsen (2007). Look-ahead control for heavy trucks to minimize trip time and fuel consumption. Accepted to 5th IFAC Symposium on Advances in Automotive Control. Monterey Coast CA, USA.

Hellström, Erik, Anders Fröberg and Lars Nielsen (2006). A real-time fuel-optimal cruise controller for heavy trucks using road topography information. number 2006-01-0008 In: $S A E$ World Congress 2006.

Kailath, T., A.H. Sayed and B. Hassibi (2000). Linear estimation. Upper Saddle River, NJ.

Kiencke, U. and L. Nielsen (2003). Automotive Control Systems. Springer Verlag, Berlin.

Lattemann, F., K. Neiss, S. Terwen and T. Connolly (2004). The predictive cruise control-a system to reduce fuel consumption of heavy duty trucks. SAE Technical Paper Series.

Lingman, P. and B. Schmidtbauer (2001). Road slope and vehicle mass estimation using Kalman filtering. In: Proceedings of the 19th IAVSD Symposium. Copenhagen, Denmark.

Schroedl, S., K. Wagstaff, S. Rogers, P. Langley and C. Wilson (2004). Mining gps traces for map refinement. Data Mining and Knowledge Discovery 9, 59-87.

Terwen, S., M. Back and V. Krebs (2004). Predictive powertrain control for heavy duty trucks. In: Proceedings of IFAC Symposium on Advances in Automotive Control. Salerno, Italy.

Vahidi, A., A. Stefanopolou and H. Peng (2005). Recursive least squares with forgetting for online estimation of vehicle mass and road grade: Theory and experiments. Journal of Vehicle System Dynamics 43, 31-57. 